\title{
A reverse Stroop effect without translation or reading difficulty
}

\author{
Chris BLaIS \\ University of California, Berkeley, California \\ AND \\ DEREK BESNER \\ University of Waterloo, Waterloo, Ontario, Canada
}

\begin{abstract}
It is well known that irrelevant color words affect the time needed to identify the color they are displayed in (the Stroop effect). One major view is that a reverse Stroop effect (RSE) - in which the irrelevant color affects the time needed to identify the word - does not occur unless a translation is needed between domain-specific memory codes. In the present article, we report an experiment in which the reverse Stroop effect was investigated by having subjects identify a colored word at fixation by pointing to a location on the screen containing that word. Although the response was untranslated, an RSE was observed. An account is provided in which the strength of association between a stimulus and a specific response plays a central role.
\end{abstract}

Stroop (1935) was the first to report that irrelevant color words influence color naming. Many hundreds of papers have subsequently explored various facets of this Stroop effect (see MacLeod's 1991 review). In contrast, relatively few researchers have explored the reverse Stroop task, in which the subject must identify the word and ignore the color in which it is displayed (see, e.g., Dunbar \& MacLeod, 1984; Stroop, 1935). The purpose of the present article is to reconsider a major account, translation, that purports to explain the conditions that produce the presence or absence of both Stroop and reverse Stroop effects. We then report a reverse Stroop task experiment that yielded a reverse Stroop effect (RSE) in the absence of a translation. We conclude by outlining a strength of association account that emphasizes the role of a specific response.

\section{A Translation Account}

Translation accounts assert that the type of response made to the target is central. These accounts assert that the irrelevant dimension affects performance when a correct response requires a translation between memory codes (see, e.g., Glaser \& Glaser, 1989; Sugg \& McDonald, 1994; Virzi \& Egeth, 1985). In these accounts, words and colors are processed in their own subsystems. The code in which each of these subsystems operates is asserted to be unique. However, when both the target and its response are considered to be represented in a common code, no translation is required. For example, no translation is needed when a word is read aloud because both the word and its response share a common code - in this case, a linguistic one. ${ }^{1}$ According to Sugg and McDonald, "the translation model predicts . . . no inhibition in untranslated word- response ... tasks [i.e., responding to the word with a word-labeled response]" (p. 648), which explains the absence of an RSE. When a color is named, however, it must be translated into a linguistic code in order to be verbalized, thus explaining the presence of a Stroop effect.

Blais and Besner (2006, Table 1) reviewed the literature describing a number of reverse Stroop task experiments. In those experiments, if the response to the word required a translation as defined above (e.g., sorting cards into a bin labeled with a color patch), an RSE was observed. If the response to the word did not require a translation (e.g., pressing a button labeled with a word), no RSE was observed (but see Blais \& Besner, 2006, for some exceptions).

In at least three other contexts, an RSE can be readily obtained even though a vocal response is required. First, giving subjects extensive practice in color naming prior to the reverse Stroop task yields an RSE (but note that it disappears soon after the task is completed; see Stroop, 1935, Experiment 3). Second, an RSE is obtained if the word is in such small print that it is hard to identify (see, e.g., Melara \& Mounts, 1993), or if the word is rendered difficult to read because it is printed upside down and backward and there are many other non-response-set items in the experiment (Dunbar \& MacLeod, 1984, Experiment 4). Third, an RSE is observed in a task-switching paradigm in which subjects switch between color and word identification, typically performing two color-naming trials followed by two word-reading trials over the course of several hundred trials (see, e.g., Wylie \& Allport, 2000).

All of these contexts have a common underlying theme: They make processing the word more difficult than the standard paradigm does. With extensive practice in color

C. Blais, cblais@berkeley.edu 
naming, subjects are biased to process the color, making it difficult for them to selectively attend to the word (Stroop, 1935). When the word is rendered difficult to read, more time is required to process it, thus affording the color an opportunity to interfere (Melara \& Mounts, 1993). Last, in the context of task switching, subjects perform two color-naming trials followed by two word-reading trials. Remembering which task to do on the current trial arguably consumes resources that could be put toward color-filtering processes. In short, no experiment has shown an RSE in the absence of a translation or some capacity-demanding manipulation.

\section{The Present Experiment}

The purpose of the present experiment was to determine whether an RSE also occurs in the absence of a translation but without a context that involves strong interactions with working memory (as in task switching). According to translation accounts, if the response labels are words rather than color patches, then no RSE should be observed because a translation is not required to correctly identify the word. Blais and Besner (2006) reported an RSE under these conditions, but their demonstration may be open to the objection that a translation (e.g., a visual image of the color associated with the key) was used. Because the key labels were obscured by the participants' fingers, they must have used a mental representation of the key labels, and it may be that this representation was color based-which would require a translation, according to such theories (although we note that subjects denied using this strategy when asked).

The present experiment therefore used a variant of Durgin's (2000) procedure, in which subjects identified the colored word at fixation by pointing to a printed response word in one of the four corners of the display. The advantage of this procedure is that the target word and the response word are always visible. The observation of an RSE in this context would not be easily explained in terms of translation.

It is important to note that both retinal resolution and cortical representation decline rapidly when stimuli are presented outside the fovea. This is especially problematic when the response locations vary on a trial-by-trial basis, since the response words may not be read easily without an eye movement. Given that any RSE independent of a translation is likely to be small in magnitude, visual search for the response label may obscure detection of the RSE. In visual search tasks, reaction time increases with retinal eccentricity (Carrasco, Evert, Chang, \& Katz, 1995). It has been shown that cortically magnifying items in the search display neutralizes this eccentricity effect (Carrasco \& Frieder, 1997). The central target word and response words were therefore equated in size using the cortical magnification factor described by Findlay and Gilchrist (2004, p. 14).

\section{METHOD}

\section{Subjects}

Thirty-two University of Waterloo undergraduates were tested individually and paid for their participation.

\section{Words at Fixation}

The stimulus set consisted of the words for four colors displayed at fixation in uppercase letters in 10-point Courier New font (ap- proximately $0.4^{\circ}$ tall and $1.1^{\circ}-2.3^{\circ}$ wide when viewed at $60 \mathrm{~cm}$ ). All possible word-color pairings occurred equally often. Each of the 20 possible stimuli (the words red, blue, yellow, and green, each presented in each of those four colors and, for neutral trials, in white) was presented 12 times each, yielding 48 congruent, 48 neutral, and 144 incongruent trials for a congruent:neutral:incongruent ratio of 20:20:60. The colors and the words were therefore not correlated. ${ }^{2}$ All trial types were randomly intermixed.

\section{Words at Response Locations}

The response word locations were presented $2.7^{\circ}$ diagonally from fixation, and the words were presented in gray lowercase 20-point Courier New font (approximately $0.8^{\circ}$ tall and $2.3^{\circ}-4.6^{\circ}$ wide when viewed at approximately $60 \mathrm{~cm}$ ). The words in the periphery were sized according to the cortical magnification factor noted earlier (Findlay \& Gilchrist, 2004, p. 14). Most importantly, the location of the response words varied randomly from trial to trial.

\section{Apparatus}

The experiment was programmed in e-Prime software running on a Pentium 4 computer with a color monitor. The six colors used and their corresponding RGB values were red $(255,0,0)$, blue $(0,0$, $255)$, green $(0,255,0)$, yellow $(255,255,0)$, gray $(170,170,170)$, and white $(255,255,255)$.

\section{Procedure}

On each trial, a single color-word combination was presented in the middle of the screen against a black background. The subjects identified the word by moving the cursor approximately $2.9^{\circ}$ diagonally from fixation to the corner of the screen in which the word's corresponding response word was displayed. Reaching the response location required less than $25 \mathrm{~mm}$ of mouse movement across the surface of the table. The position of the cursor was reset to $0.4^{\circ}$ below fixation (to prevent occlusion of the stimulus) at the beginning of each trial. Response time (RT) was measured as the time that elapsed between the onset of the target word and the passage of the cursor across the boundary of the invisible box defining each response location.

Each subject saw a different random order of 240 test trials. The experimental trials were preceded by 48 practice trials whose composition mirrored the test trials.

\section{RESULTS}

Two subjects were excluded as outliers (one with a median RT of 1,317 msec and one with an incongruent-congruent difference of $-93 \mathrm{msec}$, both of which were more than 3 standard deviations from the mean). The subjects' median RTs in each condition served as their measure of central tendency. Mean (of median) RTs and mean percentage errors are shown in Table 1 . Incongruent trials were $16 \mathrm{msec}$ slower than neutral trials $[t(29)=2.75, p<.01]$. Twenty of 30 subjects showed this effect. There was no difference between neutral trials and congruent trials $(t<1)$.

There was a trend toward more errors on incongruent trials than on neutral trials $[t(29)=1.50, p<.15]$. Nineteen of 30 subjects showed this error pattern. There was no difference between neutral and congruent trials $(t<1)$.

\section{DISCUSSION}

The experiment reported here yielded an RSE. This suggests that the account in which a translation is necessary to produce an RSE is not sufficient, given that the effect observed here likely occurred in the absence of transla- 
Table 1

Mean (of Median) Response Times (RTs, in Milliseconds) and Percentage Errors for All Conditions

\begin{tabular}{lcccccc}
\hline & \multicolumn{2}{c}{$\mathrm{RT}$} & & \multicolumn{2}{c}{$\%$ Error } \\
\cline { 2 - 3 } \cline { 5 - 6 } & $M$ & $S D$ & & $M$ & $S D$ \\
\hline Incongruent (I) & 803 & 93 & & 2.4 & 2.4 \\
Neutral (N) & 787 & 94 & & 1.7 & 2.5 \\
Congruent (C) & 787 & 96 & & 2.1 & 2.4 \\
Interference (I-N) & $16^{*}$ & 32 & & 0.7 & 2.6 \\
Facilitation (N-C) & 0 & 34 & & -0.3 & 2.2 \\
\hline
\end{tabular}

Note-Rows 4 and 5 show the $S D$ of the difference. ${ }^{*} p<.01$.

tion. It is important to note that the claim that translation plays no role in reverse Stroop (and Stroop) experiments is not being made. That claim is surely too strong, given that responding to a word using a color-labeled response or to a color using a word-labeled response produces considerably more interference than does responding to a word when it and its response label are either both words or both colors (see, e.g., Virzi \& Egeth, 1985, Experiment 1). ${ }^{3}$

To be sure, the set of conditions in which an RSE is observed is often far removed from the context (identification of a color using a vocal response) in which the standard Stroop effect is observed. ${ }^{4}$ Nonetheless, the present results need to be accommodated by any viable account of Stroop performance. The question now is whether any existing account provides a principled account of the data from both reverse Stroop and Stroop tasks.

\section{A Strength-of-Association Account}

One overarching comment is that episodic memory likely plays a strong role in reverse Stroop and Stroop tasks, given that experiments that employ these tasks use very few words and very few responses, and both words and responses are typically repeated numerous times within the experiment. We now briefly sketch an alternative to the translation account ${ }^{5}$ in which the strength of association between the target and the specific response is central. Unlike a translation account, which assumes that the response is important only in terms of whether it requires a translation or not, the strength of association account assumes that the response is important in terms of the amount of practice subjects have making it.

Like virtually all accounts of Stroop and reverse Stroop performance, we assume a word pathway and a color pathway that process words and colors, respectively. For purposes of illustration, we utilize Cohen, Dunbar, and McClelland's (1990) model. This model contains a layer of input units for colors, a layer of input units for words, a layer of response units, and a layer of hidden units that are modulated by a set of task units. The task units allow the model to respond to the appropriate dimension (word or color). In order to simulate the fact that reading words gets more practice than naming colors does, the model receives more training cycles with words than with colors. Consequently, the word pathway has stronger connections between the input and response units than does the color pathway. The trained model successfully simulates the pres- ence of a Stroop effect and the absence of a reverse Stroop effect (the standard effects when the response is vocal).

This model is combined here with an arguably important (but generally neglected) element of Logan's (1988) instance theory: the specific response to the target. The word pathway and the color pathway in Cohen et al.'s (1990) model are meant to reflect stimulus-response learning that has occurred over a person's lifetime. The information contained in these instances of S-R learning was specified by Logan (1988) as "the goal the participant was trying to attain, the stimulus encountered in pursuit of the goal, the interpretation given to the stimulus with respect to that goal, and the response made to the stimulus" (p. 495, italics added). Importantly, if the stimulus is encountered again in the context of the same goal, some proportion of the processing episodes it participated in will be retrieved. Moreover, the number of instances retrieved on any given trial is directly proportional to how similar the current context is to the context in which the episode was created.

As noted earlier, Cohen et al. (1990) have shown that when the required response is vocal, their model simulates results from both Stroop and reverse Stroop tasks. What happens when the required response is nonvocal, as when the subject points to a word?

\section{When the Response Consists of Pointing}

Our contention is that making the response consist of pointing will have two consequences. First, according to Logan's instance theory, both the color and word pathways (instances) will be less influential given their dissimilarity to the current task. That is, fewer instances within each pathway will be similar enough to the task afforded by the experiment. Computationally, a reduction in the connection weights across both the color and word pathways approximates this notion. More realistically, the reduction in connection weights should be larger for the word pathway because words, particularly color words such as yellow and blue, are not often used to point to an object, whereas colors themselves are pointed at regularly (e.g., "look at the blue house"). That is, we are more likely to point to a referent color than to a referent color word. In this limited sense, pointing to colors is more practiced than pointing to color words. In any case, the specific response called for here- using a mouse to point-is no more practiced for these words than for these colors. Hence, the standard asymmetry in which reading a word aloud is more practiced than naming a color is absent, which gives color an opportunity to interfere with a subject's selection of a response to a word.

One assumption that underlies virtually all theories of Stroop performance is the notion that responding to words is more practiced than responding to colors. This is certainly true when the response is vocal. However, in the current context - responding with a highly novel response - that asymmetry is essentially neutralized. Because both words and colors are essentially equal in terms of response strength, virtually symmetric interference is observed. This is evident in Durgin's (2000) study, in which he obtained $11 \mathrm{msec}$ of Stroop interference when subjects matched the 
color of the Stroop stimulus to a color patch that varied in location from trial to trial. We replicated this in our lab and found $23 \mathrm{msec}$ of interference and $6 \mathrm{msec}$ (n.s.) of facilitation using an untranslated color response (i.e., a Stroop task in which the subject matched the color of the Stroop word to a color patch whose location varied from trial to trial).

As an existence proof of this idea, we implemented a four-response version of the network described in Cohen et al. (1990). The connection weights were hand set to the values shown in Cohen et al.'s Figure 3. Our implementation exactly reproduced the results shown in Cohen et al.'s Figure 5B. Next, we reduced the connection weights in the word pathway to $60 \%$ of their original values and those in the color pathway to $85 \%$ of their original values to approximate the idea that pointing to color words has less in common with reading color words aloud than pointing to colors has in common with naming colors. This change was sufficient to produce reverse Stroop interference of the magnitude observed in the experiment reported here. ${ }^{6}$

\section{Conclusion}

Whether this strength of association account, which stresses the importance of the specific response, will hold up to more detailed scrutiny (e.g., in a fully implemented instance model) remains to be seen. What should no longer be ignored is the growing body of evidence that an RSE can be obtained under conditions in which translation is not likely to be in play.

\section{AUTHOR NOTE}

This research was supported by Grant A0998 from the Natural Sciences and Engineering Research Council of Canada to D.B. Correspondence concerning this article should be addressed to C. Blais, Helen Wills Neuroscience Institute, 132 Barker Hall MC 3190, University of California, Berkeley, CA 94720 (e-mail: cblais@berkeley.edu), or to D. Besner, Psychology Department, University of Waterloo, Waterloo, ON, N2L 3G1 Canada (e-mail: dbesner@watarts.uwaterloo.ca).

\section{REFERENCES}

Blais, C., \& Besner, D. (2006). Reverse Stroop effects with untranslated responses. Journal of Experimental Psychology: Human Perception \& Performance, 32, 1345-1353.

Carrasco, M., Evert, D. L., Chang, I., \& Katz, S. M. (1995). The eccentricity effect: Target eccentricity affects performance on conjunction searches. Perception \& Psychophysics, 57, 1241-1261.

CARrasco, M., \& Frieder, K. S. (1997). Cortical magnification neutralizes the eccentricity effect in visual search. Vision Research, 37, 63-82.

Cohen, J. D., Dunbar, K., \& McClelland, J. L. (1990). On the control of automatic processes: A parallel distributed account of the Stroop effect. Psychological Review, 97, 332-361.

Dishon-Berkovits, M., \& Algom, D. (2000). The Stroop effect: It is not the robust phenomenon that you have thought it to be. Memory \& Cognition, 28, 1437-1449.

DunBAR, K., \& MACLEOD, C. M. (1984). A horse race of a different color: Stroop interference patterns with transformed words. Journal of Experimental Psychology: Human Perception \& Performance, 10, 622-639.
Durgin, F. H. (2000). The reverse Stroop effect. Psychonomic Bulletin \& Review, 7, 121-125.

Findlay, J. M., \& Gilchrist, I. D. (2004). Active vision: The psychology of looking and seeing. Oxford: Oxford University Press.

Glaser, W. R., \& Glaser, M. O. (1989). Context effects in Stroop-like word and picture processing. Journal of Experimental Psychology: General, 118, 13-42.

Logan, G. D. (1988). Toward an instance theory of automatization. Psychological Review, 95, 492-527.

MACLEOD, C. M. (1991). Half a century of research on the Stroop effect: An integrative review. Psychological Bulletin, 109, 163-203.

Melara, R. D., \& Algom, D. (2003). Driven by information: A tectonic theory of Stroop effects. Psychological Review, 110, 422-471.

Melara, R. D., \& Mounts, J. R. (1993). Selective attention to Stroop dimensions: Effects of baseline discriminability, response mode, and practice. Memory \& Cognition, 21, 627-645.

STROOP, J. R. (1935). Studies of interference in serial verbal reactions. Journal of Experimental Psychology, 18, 643-662.

SugG, M. J., \& McDonald, J. E. (1994). Time course inhibition in color-response and word-response versions of the Stroop task. Journal of Experimental Psychology: Human Perception \& Performance, 20, 647-675.

VIRZI, R. A., \& Egeth, H. E. (1985). Toward a translational model of Stroop interference. Memory \& Cognition, 13, 304-319.

Wylie, G., \& Allport, A. (2000). Task switching and the measurement of "switch costs." Psychological Research, 63, 212-233.

\section{NOTES}

1. As Coltheart noted in his review of a previous version of this article, a translation between orthography and phonology occurs in reading aloud. Thus, the assertion by Virzi and Egeth (1985) and Sugg and McDonald (1994) that reading aloud does not require a translation makes their accounts difficult to understand.

2. Dishon-Berkovits and Algom (2000) claimed that a correlation between two dimensions drove interference in the Stroop task, because subjects used information about the word to predict the color, which caused them to attend to the word.

3. We have confirmed the Virzi and Egeth (1985) result using the present paradigm. Specifically, a version of our experiment was conducted in which the response labels were color patches rather than words. There was a 53-msec interference effect and a 48-msec facilitation effect (both $p$ s $<.001)$. Both of these effects are larger than the ones reported in the present experiment $(p \mathrm{~s}<.001)$.

4. Melara and Algom (2003) argued that the nature of the asymmetry in interference was a direct result of using vocal responses, since they bias word responses.

5. One can explain the present data in the context of a translation account if the color is assumed to have unconditional access to the lexicon even when a translation is not required (see Sugg \& McDonald, 1994, p. 660 and their Figure 4) in the same way that Sugg and McDonald assumed that responding to the color by pressing a color-labeled button (an untranslated response) produced interference because the word was processed and had unconditional access to semantics. The problem with this approach is that classifying a response as translated or untranslated becomes meaningless in the context of the very theory that claims that whether a response is translated or not is central to explaining performance.

6. The model still produces a small (but significant) facilitation effect that we did not observe in our human data.

(Manuscript received June 13, 2006; revision accepted for publication August 14, 2006.) 